\title{
The role of conformational diffusion in ion channel gating
}

\author{
Igor Goychuk*, Peter Hänggi \\ Universität Augsburg, Institut für Physik, Theoretische Physik I, \\ Universitätsstr. 1, D-86135 Augsburg, Germany
}

\begin{abstract}
We consider an exactly tractable model of the Kramers type for the voltage-dependent gating dynamics of single ion channels. It is assumed that the gating dynamics is caused by the thermally activated transitions in a bistable potential. Moreover, the closed state of the channel is highly degenerate and embraces the whole manifold of closed substates. Opening of the ion channel is energetically prohibited from most of the closed substates and requires a special conformation where the voltage sensor can move along an activation pathway and trigger the transition into the open conformation. When the corresponding activation barrier towards the channel's opening is removed by the applied voltage, the statistics of non-conducting time intervals become strongly influenced by the conformational diffusion. For the corresponding supra-threshold voltages, our model explains the origin of the power law distribution of the closed time intervals. The exponential-linear dependence of the opening rate on voltage, often used as an experimental fit, is also reproduced by our model.
\end{abstract}

Key words: ion channels, Kramers theory, conformational diffusion, dwelling time distributions

\section{Introduction}

Ion channels present complex protein structures embedded across the biological cell membranes thereby forming the ion-conducting, selective nanopores [1]. The conformational dynamics of these special proteins, which is known under the label of gating dynamics, results in the spontaneous openings and

* Corresponding author, tel.: +49-821-598-3235, fax:+49-821-598-3222, e-mail: goychuk@physik.uni-augsburg.de

Preprint submitted to Elsevier Science 31 October 2018 
closures of ion channels [1]. In the simplest case, this gating dynamics has the "all-or-none" character and can be symbolized by the following kinetic scheme,

$$
C \underset{k_{c}(V)}{\stackrel{k_{o}(V)}{\overleftrightarrow{\rightleftarrows}}} O
$$

Accordingly, the conductance of ion channel fluctuates stochastically between some finite value and nearly zero. In the case of voltage-dependent ion channels, like a delayed rectifier potassium channel, or a Shaker $\mathrm{K}^{+}$channel, the opening rate, $k_{o}(V)$, and the closing rate, $k_{c}(V)$, are both dependent on the applied voltage $V$.

The invention of the patch clamp technique [2] enables one to observe the current and the conductance fluctuations in real time with a resolution on the level of single ion channels. Moreover, the study of the statistics of dwelling timeintervals becomes feasible. As a matter of fact, the patch clamp experiments with single ion channels pioneered the whole area of single molecular research. The gating dynamics of an ion channel with one closed and one open states, as seen visually in the patch clamp recordings, can be characterized by the probability densities of closed, $f_{c}(t)$, and open, $f_{o}(t)$ dwelling time-intervals. The experimental studies reveal that in many ion channels the distribution of open dwelling times is approximately exponential, $f_{o}(t)=k_{c} \exp \left(-k_{c} t\right)$. However, the distribution of closed time intervals $f_{c}(t)$ frequently involves the sum of many exponentials, $\lambda_{i} \exp \left(-\lambda_{i} t\right)$, i.e.,

$$
f_{c}(t)=\sum_{i=1}^{N} c_{i} \lambda_{i} \exp \left(-\lambda_{i} t\right),
$$

with weights $c_{i}$,obeying $\sum_{i=1}^{N} c_{i}=1$. The rationale behind this fitting procedure is the assumption that the closed state consists of $N$ kinetically distinct discrete substates separated by large potential barriers. The gating dynamics is then captured by a discrete Markovian kinetic scheme with the rate constants determined by the Arrhenius law.

Such an approach presents nowadays the standard in the neurophysiology [3]. An essential drawback of it is, however, that the number of closed substates needed for agreement with the experimental data can depend on the range of applied voltages and temperatures used in the experiment. For example, the experimental gating dynamics of a Shaker potassium channel has been reasonably described by a sequential 8-state Markovian scheme with 7 closed states for a temperature about $T=20{ }^{\circ} C$ [4]. However, when the same research group extended later on their model to embrace the small range of temperatures $10-20{ }^{\circ} \mathrm{C}$, three additional closed states have been introduced [5]. This 
"proliferation" of the number of discrete states, required for the consistent description of experimental data, is rarely addressed within the approach based on a discrete Markovian description.

Moreover, it may happen that for some channels the closed time distribution can be more conveniently fitted by a stretched exponential [6], or by a power law dependence $[7,8,9]$ with a few parameters only. This observation gave rise to the so-called fractal models of the ion channel gating dynamics such as put forward, by example, by Liebovitch et al. [6]. The diffusion models introduced by Millhauser et al. [7], Läuger [10] and Condat and Jäckle [11] are intrinsically based on the concept of dynamical conformational substates in proteins, an idea which has been pioneered by Frauenfelder et al. [12]. The diffusion models serve as a microscopic justification for the fractal modeling. On the other hand, the discrete diffusion models yet present Markovian models with a large number of states. The non-Markovian, fractal behavior emerges from the projection of a full Markovian dynamics onto the subspace of two observable states as symbolized by the kinetic scheme (1).

Alternatively, the discrete diffusion can be replaced by the continuous diffusion on a potential landscape. Then, the distinct minima of this landscape, separated by substantial potential barriers, correspond to the discrete states in the mainstream approach. However, it may happen that, depending on the applied voltage, some of the distinct barriers disappear. Then, the new features of gating dynamics come into the play, which are inevitably missed in the discrete modeling. It seems therefore that a compromise between the discrete Markovian schemes and a continuous conformational diffusion approach is called for $[13,14]$. Especially, the continuous diffusion models, if set up sufficiently simple to allow an analytic treatment, are capable to provide a new insight into the problem of ion channel gating. In the present work, we refine and justify further the approach put forward in Ref. [14].

\section{Model of gating dynamics}

To start, let us consider the model depicted in Fig. 1. Its central element is the voltage-dependent transitions in a bistable, piecewise linear potential along the $x$-axis, see l.h.s. in Fig. 1 . This bistable potential corresponds to the motion of the so-called voltage sensor. The voltage sensor presents a functional part of the ion channel machinery which changes its position in response to the changes in the transmembrane potential. In accord with the current view [1], the voltage sensor is formed by the system of four positively charged S4 $\alpha$-helices which carry a total gating charge $q \sim 10-13 \mathrm{e}_{0}$, where $e_{0}$ is the positively-valued, elementary charge. When $x=0$, the voltage sensor is in its resting position; when $x=x_{0}$, the voltage sensor is fully activated and the 


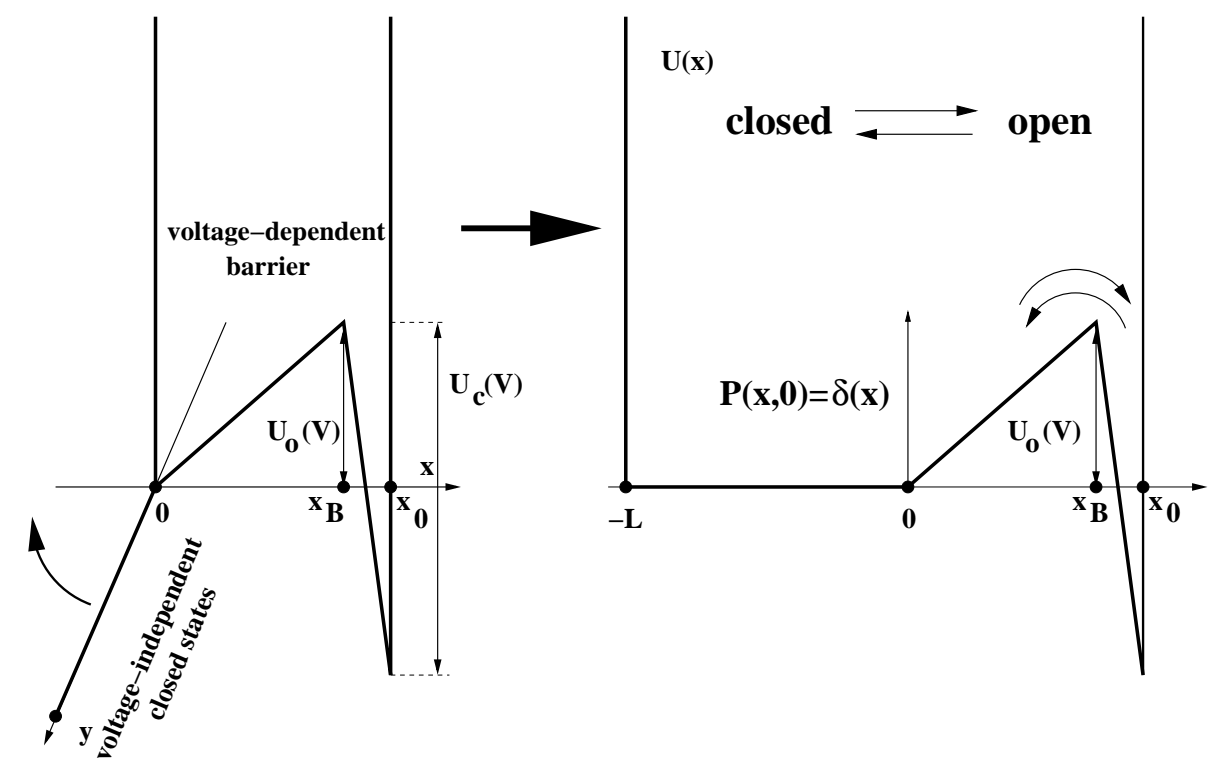

Fig. 1. Sketch of the studied model. The left part of figure involves the two-dimensional conformational space for the gating dynamics. The $x$-axis corresponds to the voltage sensor degrees of freedom and the $y$-axis to the conformational diffusion. Note that only the $y=0$ cross-section, $U(x, 0)$, of the two-dimensional conformational potential $U(x, y)$ possesses a bistable character. For $y \geq \delta$ exceeding some small $\delta$-vicinity of $y=0$, the $y=a=$ constant cross-sections, $U(x, a)$, are essentially monostable, exhibiting sharp minima at $x=0$. Moreover, the potential $U(0, y)$ is flat in the $y$-direction. These features allow one to simplify the picture of a two-dimensional reaction state space to the case of one-dimensional reaction coordinate (see the right part in the figure) as described in the text.

complete gating charge is moved across the membrane. Moreover, the drop $V$ of the transmembrane electric potential is assumed to occur linearly on the characteristic length $x_{0}$ which corresponds to the effective width of the cellular membrane within the region of the gating charge translocation. Then, the energy barriers, $U_{o}(V)$ and $U_{c}(V)$, follow as

$$
\begin{aligned}
& U_{o}(V)=q r\left(V_{c}-V\right), \\
& U_{c}(V)=U_{c}^{(0)}-q(1-r)\left(V_{c}-V\right),
\end{aligned}
$$

where $r:=x_{B} / x_{0}$. Furthermore, $V_{c}$ in (3) corresponds to the threshold value of the voltage $V$ when the activation barrier towards the channel opening vanishes and $U_{c}^{(0)}$ in (4) is the corresponding height of the activation barrier towards the channel closing. Note that for $V>V_{c}, U_{o}(V)$ is negative, but $U_{c}(V)$ has always a large positive value, since one assumes that $U_{c}^{(0)}$ is large and $1-r<<1$. These assumptions correspond to the experimental observations that the closing rate has a very strong dependence on the temperature and it is less voltage-sensitive than the opening rate [1]. Furthermore, one assumes that the voltage sensor located at the position $x>x_{B}\left(x_{B}\right.$ corresponds 
to the top of the activation barrier for the sub-threshold voltages, $V<V_{c}$ ) triggers the conformation change in the activation gate. This latter conformational change then leads to the channel opening. It is worth to notice that in accord with the reasoning in $[15,16]$, the activation gate is likely formed by the bundle of the intracellular ends of inner, pore-lining S6 $\alpha$-helices [1]. The motion of the voltage sensor creates an indirect mechanical torque on the activation gate. This torque is mediated by other structural elements of the channel protein. For this reason, the link between the voltage sensor and the activation gate may be flexible and this circumstance can introduce a kind of mechanical hysteresis. Namely, the closure of the activation gate does not occur immediately, when the voltage sensor crosses the border at $x=x_{B}$ in the back direction, but rather requires that (is most probable when) the voltage sensor is fully returned to its resting position at $x=0$. For this reason, by calculating the dwelling time distribution $f_{c}(t)$ we assume the initial condition for the probability density $P(x, t)$ in the form $P(x, 0)=\delta(x)$, i.e., each and every closing time-interval starts when the voltage sensor has fully returned. This inevitably presents an approximation to the reality. In accord with the general activation theory of Kramers [17], we also have to impose an absorbing boundary at $x>x_{B}$. The most natural choice for this is $x=x_{0}$. However, in view of our model assumption $U_{c}^{(0)} \gg k_{B} T$ one can safely move the absorbing boundary to $x=x_{B}$, i.e., $P\left(x_{B}, t\right)=0$ at all times $t \geq 0$. The reason for this is that the final transition $x_{B} \rightarrow x_{0}$ leading to the opening is very quick. The same is assumed in many discrete Markovian schemes [1]. Given this assumption, the duration of this final step is negligible in calculating $f_{c}(t)$. Furthermore, in order to find the distribution of open times, $f_{o}(t)$, one should put the reflecting boundary at $x=x_{0}$ and the sink (absorbing boundary) at $x=0$, in accord with the above discussion.

The next important element of our model is the assumption that the voltage sensor can operate only if the protein acquires a special pre-open conformation. Otherwise, its motion is energetically prohibited due to the steric factors. To account for this type of behavior we introduce the additional, "perpendicular" conformational reaction coordinate $y$, see 1.h.s. in Fig. 1. It describes the conformational diffusion. One assumes that the corresponding dynamics is only weakly voltage-dependent and we shall neglect the accompanying redistribution of charges in the protein molecule. Actually, this conformational diffusion should occur in a hierarchically arranged, ultrametric conformational space $[12,18,19]$. In a simplified manner, one can model this hierarchical features by a random walk with the mean-squared displacement $\left\langle\Delta y^{2}(t)\right\rangle \propto t^{\gamma}$. This latter relation holds, strictly speaking, if the diffusion is unbounded in space. The bounded character, however, is important for the following. We restrict ourselves in this paper to the case of normal diffusion, $\gamma=1$, as the simplest possibility. Finally, within this outlined picture one can "convert" the dynamics along the $y$-axis into the dynamics along the $x$-axis, by use of the extra part $[-L, 0]$ there, see r.h.s in Fig. 1, whereby introducing the aux- 
iliary diffusion length $L$ and the diffusion coefficient $D=k_{B} T / \eta$. Here, $\eta$ is the viscous friction coefficient characterizing the conformational dynamics. In order to account for the bounded character of the conformational diffusion, we impose the reflecting boundary condition $\left.\frac{\partial P(x, t)}{\partial x}\right|_{x=-L}=0$ at $x=-L$ for all $t$. Note that $\eta$ should generally depend exponentially on the temperature, i.e.

$$
\eta=\eta_{0} \exp \left(\epsilon / k_{B} T\right)
$$

where $\epsilon$ is a characteristic activation energy for transitions between conformational microstates. It can be in the range of several $k_{B} T_{\text {room }}$. The parameters $D$ and $L$ are conveniently combined into the single parameter - the conformational diffusion time, i.e.,

$$
\tau_{D}=L^{2} / D \propto \exp \left(\epsilon / k_{B} T\right) / k_{B} T
$$

which constitutes an important parameter of the theory.

\section{Theory of ion channel gating}

In order to find $f_{c}(t)$ one has to solve the Smoluchowski dynamics

$$
\frac{\partial P(x, t)}{\partial t}=D \frac{\partial}{\partial x}\left(\frac{\partial}{\partial x}+\beta \frac{\partial U(x)}{\partial x}\right) P(x, t),
$$

where $\beta=1 /\left(k_{B} T\right)$, supplemented by the initial and the boundary conditions of reflection and absorption discussed above. The closed residence time distribution then follows as

$$
f_{c}(t)=-\frac{d \Phi_{c}(t)}{d t}
$$

where $\Phi_{c}(t)=\int_{-L}^{x_{B}} P(x, t) d x$ is the survival probability in the closed state.

By use of the standard Laplace transform method we arrive at the following exact solution for the Laplace-transformed distribution of closed times $\tilde{f}_{c}(s)$, see in Ref. [14]:

$$
\tilde{f}_{c}(s)=\frac{A(s)}{B(s)}
$$

where 


$$
\begin{aligned}
A(s) & =\exp \left(-\beta U_{o}(V) / 2\right) \sqrt{\beta^{2} U_{o}^{2}(V)+4 \xi^{2} \tau_{D} s} \\
B(s) & =\sqrt{\beta^{2} U_{o}^{2}(V)+4 \xi^{2} \tau_{D} s} \cosh \left(\sqrt{\beta^{2} U_{o}^{2}(V)+4 \xi^{2} \tau_{D} s} / 2\right) \\
& +\left(2 \xi \sqrt{\tau_{D} s} \tanh \sqrt{\tau_{D} s}-\beta U_{o}(V)\right) \sinh \left(\sqrt{\beta^{2} U_{o}^{2}(V)+4 \xi^{2} \tau_{D} s} / 2\right)
\end{aligned}
$$

where the parameter $\xi$ is given by $\xi:=x_{B} / L$. The explicit result in Eqs. (8)(10) allows one to find all moments of the closed residence time distribution. In particular, the mean closed residence time,

$$
\left\langle T_{c}\right\rangle:=\int_{0}^{\infty} t f_{c}(t) d t=\lim _{s \rightarrow 0}\left[1-\tilde{f}_{c}(s)\right] / s
$$

emerges as

$$
\left\langle T_{c}(V)\right\rangle=\tau_{D} \xi \frac{\beta U_{o}(V)\left(e^{\beta U_{o}(V)}-1-\xi\right)+\xi\left(e^{\beta U_{o}(V)}-1\right)}{\beta^{2} U_{o}^{2}(V)} .
$$

The effective opening rate can be defined as $k_{o}(V):=1 /\left\langle T_{c}(V)\right\rangle$. Let us consider the limiting case $\xi=x_{B} / L \ll 1$. In the language of discrete substates, this limit is tantamount to the assumption that the number of quasidegenerate conformational substates, which correspond to the resting position of the voltage sensor, largely exceeds that of the voltage-sensor. Under this assumption, we obtain in leading order of $\xi$

$$
k_{o}(V)=\frac{1}{\left\langle T_{c}\right\rangle} \approx \frac{1}{\xi \tau_{D}} \frac{\beta r q\left(V-V_{c}\right)}{1-\exp \left[-\beta r q\left(V-V_{c}\right)\right]} .
$$

Note that the functional form in Eq. (12) is nothing but the familiar exponentiallinear dependence used as a phenomenological experimental fit in the celebrated paper by Hodgkin and Huxley [20] used to describe the voltagedependence of the opening rate of a single gate in the potassium channels. This form is commonly used to parameterize the opening rate of the potassium channels, see, e. g., in [21]. Our model provides a detailed justification for this experimental result. Its remarkable feature is that the dependence of the rate on voltage is exponential for $V<V_{c}$, when the energy barrier towards activation of the voltage sensor is essential, $k_{B} T<U_{o}(V)$. This exponential voltage-dependence implies in virtue of (5) an exponential dependence on temperature as well, i.e.,

$$
k_{o} \propto \exp \left\{-\left[\epsilon+r q\left(V_{c}-V\right)\right] / k_{B} T\right\}
$$

This exponential temperature dependence has two sources: a first one is due to the activation barrier of the voltage sensor $U_{o}(V)$, while a second one is due to the activation barrier $\epsilon$ between diffusional microstates, which we have assumed for reasons of simplicity to be of equal height. The barrier $U_{o}(V)$ 


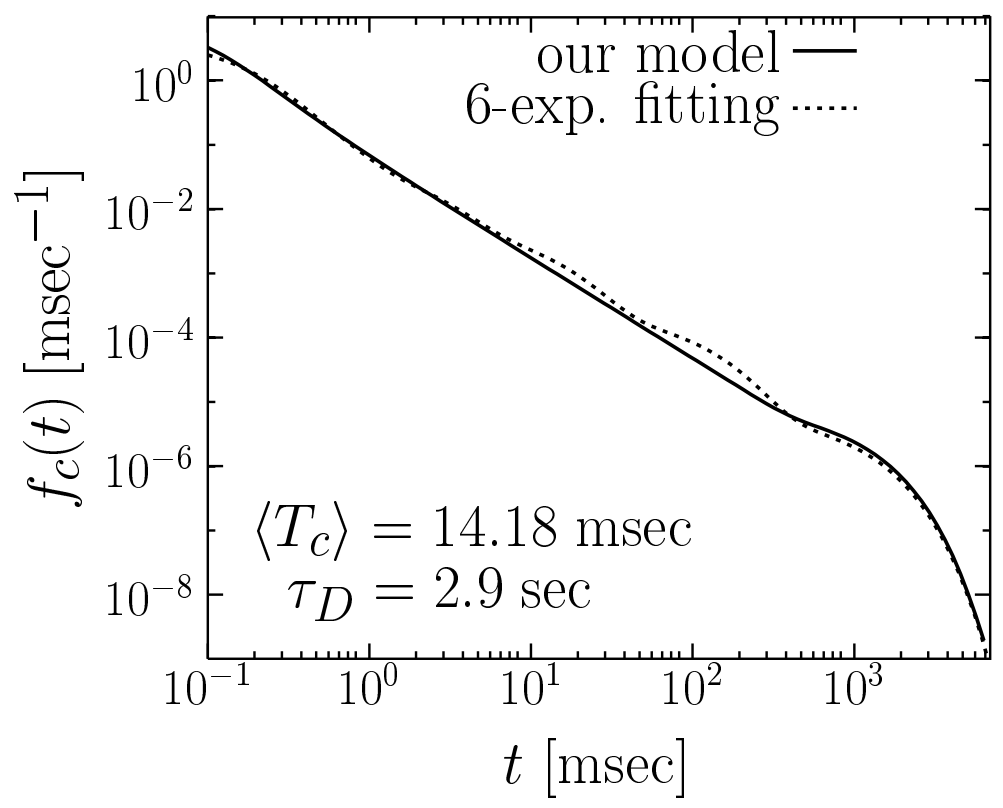

Fig. 2. Closed time probability density from our model, Eq. (7), (solid line) and 6-exponential fitting procedure of the experimental data from Ref. [8] (dotted line). The following set of parameters is used in our calculations: $\tau_{D}=2.9 \mathrm{sec}, \xi=0.01$ and $\beta U_{o}(V)=-1.653$.

can acquire large values. For example, assuming typical values $V_{c}=-40$ $\mathrm{meV}, r q=10 e_{0}$ and a room temperature $k_{B} T_{\text {room }}=25 \mathrm{meV}$ one obtains $U_{o}\left(V_{r}\right)=20 k_{B} T$ for the cell resting potential $V_{r}=-90 \mathrm{meV}$. Furthermore, when the activation barrier $U_{o}(V)$ vanishes for $V>V_{c}$, it follows from (12) that the rate $k_{o}(V)$ exhibits the linear dependence on voltage, i.e., $k_{o}(V) \propto\left(V-V_{c}\right)$. In this case, its temperature dependence is distinctly reduced and becomes mainly determined by the activation barrier $\epsilon$ of the conformational diffusion. The latter one can assume a few $k_{B} T_{\text {room }}$ only. The very different temperature dependences of the opening rate for $V \ll V_{c}$ and for $V>V_{c}$ present an interesting feature of our model which calls for an experimental verification.

It is worthwhile to notice that the discussed crossover from an exponential to linear voltage dependence of the opening rate is qualitatively preserved for any value of $\xi$, including the extreme case $\xi \gg 1$. In this case $L \ll x_{B}$ and the conformational diffusion does not play a dominant role. The discussed feature is caused by the vanishing of the activation barrier $U_{o}(V)$ for $V>V_{c}$. However, the distribution of closed time-intervals $f_{c}(t)$ depends qualitatively on $\xi$. Namely, for $\xi \ll 1$ and $V>V_{c}$ it displays a power law regime, i.e.

$$
f_{c}(t) \approx \frac{1}{2\left(\pi \tau_{D}\right)^{1 / 2} k_{o} t^{3 / 2}}
$$

for the intermediate time scale $\left\langle T_{c}\right\rangle^{2} / \tau_{D} \ll t \ll \tau_{D}$. In Fig. 2, we illustrate this prominent feature for the following model parameters: $\tau_{D}=2.9 \mathrm{sec}, \xi=0.01$ 


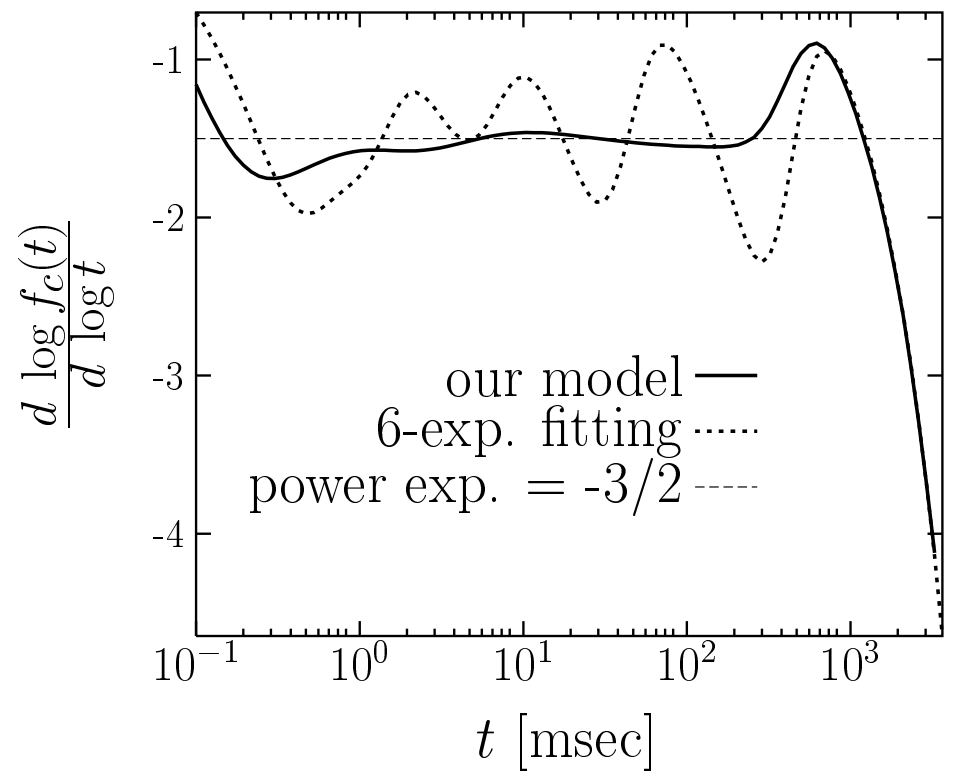

Fig. 3. Logarithmic derivative of the closed time probability density $f_{c}(t)$. The solid line and the dotted line correspond to those in Fig. 2; the long-dashed level line corresponds to strict power law dependence $f_{c}(t) \propto t^{-3 / 2}$. The oscillating behavior reveals the hierarchical character of the conformational dynamics.

and $\beta U_{o}(V)=-1.653$. The solid line denotes the closed time probability density obtained from our model, Eqs. (8)-(10), by a numerical inversion of the Laplace transform $\tilde{f}_{c}(s)$. The short-dashed line presents the fitting of the experimental data for the delayed rectifier $\mathrm{K}^{+}$channel from a neuroblastoma $\times$ glioma cell by use of Eq. (2) with 6 exponentials. This fitting procedure is taken from Ref. [8] (see, Table 4 therein) and implicitly corresponds to a discrete Markovian scheme with 6 closed substates. Both results describe well the intermediate power law trend and the exponential tail of the closed time-interval distribution. Nevertheless, some small distinctions can be distinguished.

The plot of the logarithmic derivative, $d \log f_{c}(t) / d \log t$, versus the logarithm of time $t$ in Fig. 3 renders these distinctions much more visible. The advantage of such a representation as in Fig. 3 is - in accordance with the reasoning in Ref. [19] - that the hierarchical, tree-like relaxation dynamics reveals itself by logarithmic oscillations around the level line that corresponds to the power law trend. Remarkably enough, the exponential fit from Ref. [8] does exhibit such logarithmic oscillations, cf. Fig. 3. Thus, this result seems to support the hierarchical character of the conformational dynamics. Our simplified model does not distinctly display these fine features as these are rooted in the discrete nature of hierarchical states. Nevertheless, the power law trend, which reveals the presence and the importance of the conformational dynamics, is reproduced by our model. Moreover, its appealing feature is that it requires only few parameters which possess a clear physical meaning. The particular 
value of the power law exponent $-3 / 2$ corresponds to the conformational dynamics modeled as a bounded normal diffusion. Other power law exponents, also seen experimentally [9], require a generalization of our model to the case of anomalous diffusion. Such corresponding work is presently in progress.

\section{Summary and conclusions}

We herewith have presented a simple model of the complex gating dynamics in voltage-dependent potassium ion channels. It is based on the concept of conformational diffusion. In particular, we assumed that the conformational change leading to the opening of ion channel is triggered by the voltage sensor which, in its turn, is permitted only when the channel protein acquires a special pre-open configuration. When the ion channel is closed, it exhibits an internal, conformational diffusion over the manifold of conformational substates which do not possess a sensitive voltage dependence. In a simplified manner, this conformational diffusion has been mathematically modeled by bounded normal diffusion. Moreover, it has been assumed that the open conformation of the channel is more ordered, with less conformational substates. Then, the conformational diffusion does not play an essential role. In the language of statistical thermodynamics this means that the ion channel upon opening undergoes a kind of ordering transition into a state with lower configurational entropy.

We should also stress here that our simple model is aimed not to replace the standard discrete Markovian modeling [3], but rather to complement these efforts by highlighting some basic physical features which otherwise become blurred with the standard approach. In particular, it has been shown that the transition from an exponential to linear voltage dependence of the opening rate occurs when the activation barrier for the voltage sensor towards the channel's opening vanishes due to the applied transmembrane voltage. Moreover, if the conformational diffusion time $\tau_{D}$ exceeds largely the mean duration of closed time intervals $\left\langle T_{c}\right\rangle$, the closed time distribution exhibits a power law feature on the intermediate time scale $\left\langle T_{c}\right\rangle^{2} / \tau_{D} \ll t \ll \tau_{D}$. This power law changes over into an exponential tail for times $t>\tau_{D}$. These features are seemingly consistent with the experimental observations for some $\mathrm{K}^{+}$-channels. The true physical benchmark of our model is, however, the prediction that the opening rate will become much less temperature-dependent for supra-threshold voltages. The weak temperature dependence in this latter regime should correlate with a weak voltage dependence. This distinct prediction calls for an experimental verification, which hopefully will support our reasoning for the gating dynamics in ion channels.

Acknowledgments. This work has been supported by the Deutsche Forschungs- 
gemeinschaft via the Sonderforschungsbereich SFB-486, Manipulation of matter on the nanoscale.

\section{References}

[1] B. Hille Ionic Channels of Excitable Membranes, 3d ed. Sinauer Associates, Sunderland, 2001.

[2] Single-Channel Recording, 2nd ed., B. Sakmann, N. Neher, Eds. Plenum, New York, 1995.

[3] D. Colquhoun, A.G. Hawkes, in [2], pp. 397-482.

[4] F. Bezanilla, E. Perozo, E. Stefani, Biophys. J. 66 (1994) 1011.

[5] B. M. Rodriguez, D. Sigg, F. Bezanilla, J. Gen. Physiol. 112 (1998) 223.

[6] L. S. Liebovitch, J. Fishbarg, J. P. Koniarek, Math. Biosci. 84 (1987) 37.

[7] G. L. Millhauser, E. E. Salpeter, R. E. Oswald, Proc. Natl. Acad. Sci. USA $\mathbf{8 5}$ (1988) 1503.

[8] M. S. P. Sansom, F. G. Ball, C. J. Kerry, R. McGee, R. L. Ramsey, P. N. R. Usherwood, Biophys. J. 56 (1989) 1229.

[9] A. L. Blatz, K. L. Magleby, J. Physiol. (London) 378 (1986) 141; A. Ring, Biochim. Biophys. Acta 856 (1986) 646; S. Mercik, K. Weron, Phys. Rev. E 63 (2001) 051910.

[10] P. Läuger, Biophys. J. 53 (1988) 877.

[11] C. A. Condat, J. Jäckle, Biophys. J. 55 (1989) 915.

[12] H. Frauenfelder, S. G. Sligar, P. G. Wolynes, Science 254 (1991) 1598; H. Frauenfelder, B. H. McMahon, Ann. Phys. (Leipzig) 9 (2000) 655.

[13] D. Sigg, H. Qian, F. Bezanilla, Biophys. J. 76 (1999) 782.

[14] I. Goychuk, P. Hänggi, Proc. Natl. Acad. Sci. USA 99 (2002) 3552.

[15] Y. Jiang, A. Lee, J. Chen, M. Cadene, B. T. Chait, R. MacKinnon, Nature (London) $\mathbf{4 1 7}$ (2002) 523.

[16] G. Yellen, Nature (London) 419 (2002) 35.

[17] P. Hänggi, P. Talkner, M. Borkovec, Rev. Mod. Phys. 62 (1990) 251.

[18] C. M. Dobson, A. Sali, and M. Karplus, Angew. Chem. Int. Ed. 37 (1998) 868.

[19] R. Metzler, J. Klafter, J. Jortner, Proc. Natl. Acad. Sci. USA 96 (1999) 11085.

[20] A.L. Hodgkin, A. F. Huxley, J. Physiol. (London) 117 (1952) 500.

[21] H. Salman, Y. Soen, and E. Braun, Phys. Rev. Lett. 21 (1996) 4458; Z. F. Mainen, J. Joerges, J. R. Huguenard, T. J. Sejnowski, Neuron 15 (1995) 1425. 